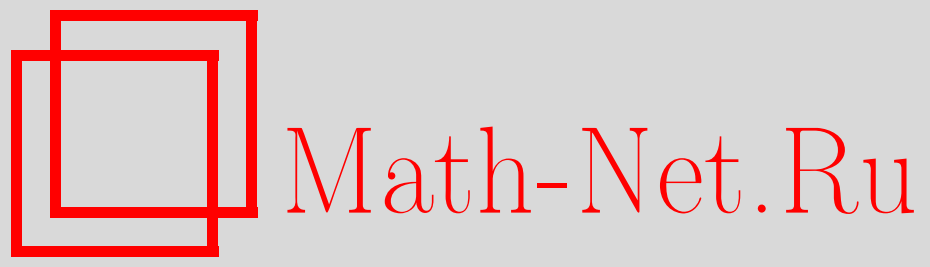

А. Оноприенко, Геометрия клетчатой бумаги, Квант, 2018, номер 11, 27-30

DOI: https://doi.org/10.4213/kvant20181103

Использование Общероссийского математического портала Math-Net.Ru подразумевает, что вы прочитали и согласны с пользовательским соглашением http://www.mathnet.ru/rus/agreement

Параметры загрузки:

IP: 3.81 .55 .215

26 апреля 2023 г., 15:54:24

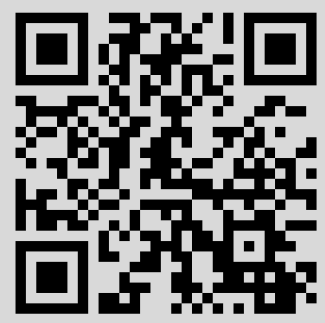




\section{Геометрия клетчатой бумаги}

\section{А.ОНОПРИЕНКО}

$\mathrm{M}$ Ы РАССКАЖЕМ ОБ ОДНОМ ИНТЕресном сюжете, подготовленном по материалам математических кружков при Московском педагогическом государственном университете (МПГУ). ${ }^{1}$

Обычный листочек в клетку таит в себе много неожиданностей. Мы обсудим несколько задач, построения в которых производятся на клетчатой плоскости. Как и в стандартных задачах на построение, можно пользоваться линейкой без делений, чтобы проводить прямые, но циркуль нам совершенно не понадобится. Как правило, именно циркуль, а не линейка, позволяет в задачах на построение «измерять» отрезки (не находить их длину, а строить отрезок, равный данному, например). Здесь же измерительным инструментом является сама плоскость!

Заглавными буквами $A, B, C, \ldots$ во всех задачах обозначены узлы клетчатой бумаги. Сторону одной клетки принимаем за 1. Начнем наше путешествие с одной простой задачи-исследования.

Задача 1. а) На клетчатой бумаге проведен отрезок $А В$, не проходящий по сторонам клеток, и отмечена точка $C$. Отметьте узел $D$ такой, ито $A B=C D$. б) Постройте все такие отрезки. Сколько их получилось?

На рисунке 1 представлены разные варианты отрезков $A B$. Постарайтесь решить задачу для каждого из этих вариантов. Точку $C$ можете выбирать в каком угодно узле (из нашего решения будет следовать, что ее расположение не важно)

1 Кружки открыты с 2016 года, а в этом году они получили поддержку Департамента образования города Москвы в рамках мероприятия «Детский университет МПГУ (организация работы кружков для московских школьников на базе МПгу)». Более подробная информация о кружках есть на сайте mathcircles.mpgu.org

DOI: https://doi.org/10.4213/kvant20181103

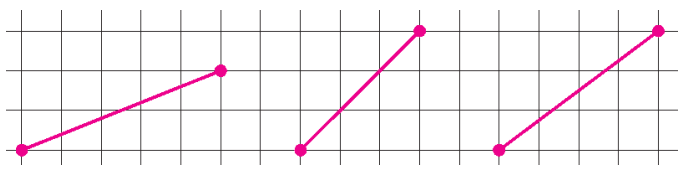

PnC. 1

Решение. а) Построим прямоугольный треугольник по сторонам сетки, одна из сторон которого - отрезок $A B$ (вообще говоря, это можно сделать двумя способами, но зафиксируем один из них). Затем перенесем этот треугольник в точку $C$. Та сторона этого треугольника, которая не идет по сторонам сетки, будет равна отрезку $A B$, так как треугольники равны по двум катетам. Это и будет искомый отрезок $C D$ (рис. $2, a)$.

Это же решение годится для любого другого отрезка $A B$.

б) Вообще говоря, в пункте а) можно было бы не строить треугольник, а внимательно рассмотреть отрезок $A B$. Для того чтобы попасть в точку $B$, мы должны от точки $A$ пройти на 5 клеток вправо и на 2 клетки вверх (эти числа приведены для первого отрезка, для других отрезков количество клеток будет другим). Если пройти от точки $C$ на 5 клеток в одном направлении и на 2 клетки в перпендикулярном

a)

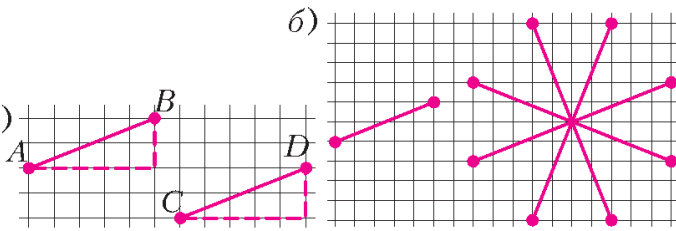

8 отрезков

в)

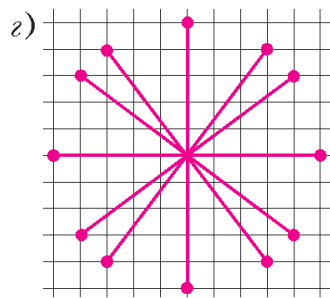

12 отрезков

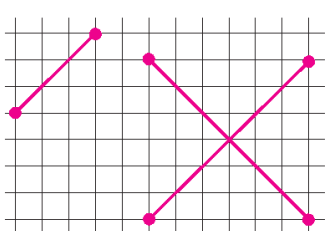

4 отрезка

Pис. 2 
изначальному направлении, то все отрезки, которые мы при этом получим, будут равны $A B$. В общем случае мы проходим $n$ клеток в одном направлении и $m$ клеток в перпендикулярном ему. Всего получается 8 отрезков: проходим $n$ клеток в одном из четырех направлений вниз-влево-вверхвправо и далее проходим $m$ клеток в одном из двух перпендикулярных направлений (рис.2,б). При $n=m$ (когда отрезок $A B$ проходит под углом $45^{\circ}$ к линиям сетки) равных отрезков получится не 8, а 4 штуки (из исходных 8 отрезков каждая соответствующая пара будет «сливаться» в один отрезок; рис.2,в).

Третий случай на рисунке 1 отличается от первых двух тем, что помимо указанных ранее восьми отрезков можно построить еще четыре. Если вспомнить теорему Пифагора, то окажется, что $4^{2}+3^{2}=5^{2}$, т.е. длина третьего отрезка равна 5. Поэтому в данном случае добавится еще 4 отрезка, идущих по сторонам клеток (рис.2,乙).

Обратите внимание, что на рисунке 2 приведены все возможные отрезки, равные исходным. Действительно, опять применим теорему Пифагора и заметим, что числа $5^{2}+2^{2}=29,3^{2}+3^{2}=18$ и $4^{2}+3^{2}=$ $=25$ не представляются по-другому в виде суммы двух квадратов натуральных чисел.

Замечание. Вообще говоря, отрезков может быть больше двенадцати, если число представимо в виде суммы двух квадратов более чем одним способом. Например, шестнадцать (скажем, $65=1^{2}+8^{2}=4^{2}+7^{2}$ - из одной точки выходят два пучка по 8 отрезков, и все они равны друг другу), двадцать (к примеру, $65^{2}=16^{2}+63^{2}=$ $=33^{2}+56^{2}-$ помимо двух пучков из 8 отрезков добавляется 4 отрезка, идущих по сторонам клеток) и т.д.

Задача 2. Незнайка нарисовал на клетчатой бумаге квадрат ABCD с вершинами в узлах сетки, стороны которого не проходят по сторонам клеток, а потом

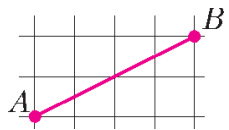

Pис. 3 все стер, оставив только точки А и В (рис.3). Восстановите рисунок Незнайки. Сколько решений имеет задача? a)

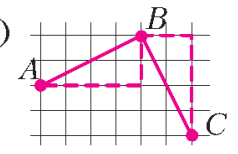

Pnc. 4

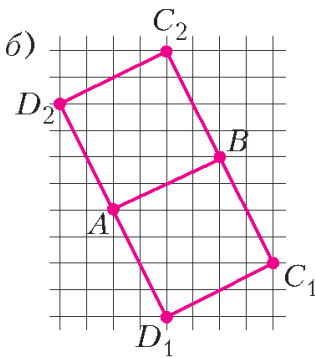

Сначала необходимо научиться строить перпендикуляр к отрезку. Как в предыдущей задаче, строим прямоугольный треугольник, одной из сторон которого будет отрезок $A B$, а затем поворачиваем его на угол $90^{\circ}$ (рис.4,a). Если посмотреть на клеточки, то мы увидим, что точка $B$ из точки $A$ получается так: надо пройти 4 клеточки вправо и 2 клеточки вверх, а точка $C$ получается из точки $B$ так: надо пройти те же самые расстояния, но в направлениях, перпендикулярных исходным, т.е. 2 клеточки вправо и 4 клеточки вниз. Далее строим перпендикуляры еще два раза, каждый раз к новой стороне. Задача имеет два решения, так как первый отрезок $B C$ можно было построить двумя способами (рис.4,б).

Мы разобрали решение задачи на конкретном примере. Нетрудно понять, что в других случаях происходит то же самое.

Задача 3. Постройте середину отрезка $A B$, не идущего по сторонам клеток.

В некоторых случаях построить середину отрезка на клетчатой бумаге очень легко. Рассмотрим отрезок $A B$ : пусть от одного его конца до другого необходимо пройти $a$ клеток вправо или влево и $b$ клеток вверх или вниз. Если оба числа $a$ и $b$ четные, то середина $A B$ попадает в узел $($ рис.5, $a)$, от которого до каждого конца отрезка необходимо пройти $\frac{a}{2}$ клеток в одном направлении и $\frac{b}{2}$ в другом.

Если же одно из чисел $a$ и $b$ четное, а другое нет, пусть четным является число $a$, то середину отрезка $A B$ можно найти, пройдя $\frac{a}{2}$ клеток вправо (или влево) и найдя пересечение отрезка с этой вертикальной линией сетки (рис.5,б). 


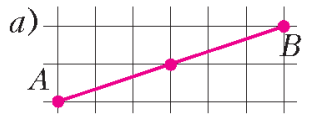

B)

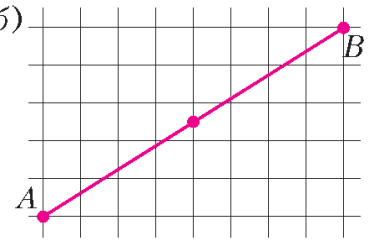

РИс. 5

Если оба числа $a$ и $b$ нечетные, то поступим следующим образом (рис.5, 8 ). Отрезок $A B$ является диагональю прямоугольника $a \times b \quad$ с вершинами в узлах сетки. Проведя вторую диагональ и найдя точку пересечения с отрезком $A B$, получим искомую середину.

Заметим, что этот метод годится для любых чисел $a$ и $b$, а не только нечетных.

Подумайте дополнительно, как построить середину отрезка, идущего по сторонам клеток. (Если длина этого отрезка четная, то середина просто попадает в узел. А если нечетная?)

Перейдем теперь к подсчету площадей на клетчатой бумаге. ${ }^{2}$

Задача 4. Найдите плошадь треугольников, изображенных на рисунке 6. Подумайте, как это сделать для произвольных треугольников с вершинами в узлах сетки.

a) Достроим прямоугольный треугольник до прямоугольника (рис.7,a). Если a)

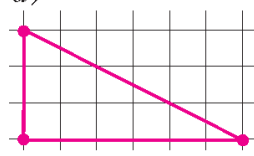

2)

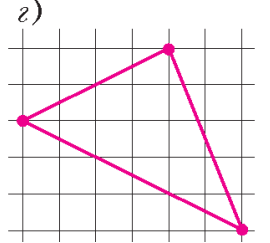

б)

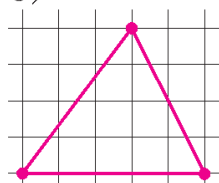

д)

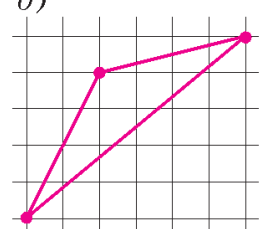

Pис. 6

${ }^{2}$ В общем случае для вычисления площади многоугольника с вершинами в узлах клетчатой бумаги можно использовать формулу Пика (см. например, статью Г.Мерзона «Формула Пика и тающий лед» в «Кванте» №9 за 2018 г.). - Прим. ред.

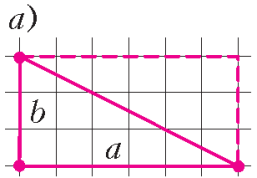

б)

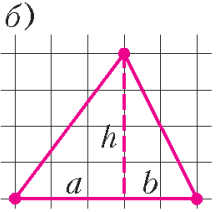

8)

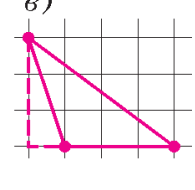

8)

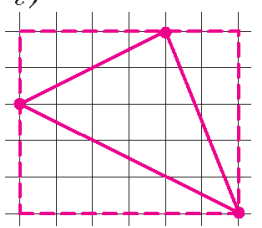

д)

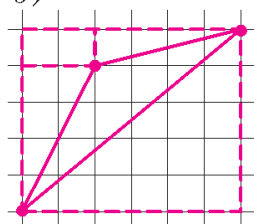

Pnc. 7

$a$ и $b$ - стороны прямоугольника, то его площадь равна $a \cdot b$, а площадь данного прямоугольного треугольника равна $\frac{a \cdot b}{2}=\frac{6 \cdot 3}{2}=9$.

б), в) Проведем высоту в треугольнике, падающую на сторону, проходящую по сторонам клеток, или на ее продолжение (рис.7,б, в). Площадь нашего треугольника равна сумме либо разности площадей двух прямоугольных треугольников (в зависимости от того, падает высота на сторону треугольника или же на ее продолжение). Проведем подсчеты. Для случая б) (сумма площадей) площадь треугольника равна $\frac{3 \cdot 4}{2}+\frac{2 \cdot 4}{2}=10$, для случая в) (разность площадей) площадь треугольника равна $\frac{4 \cdot 3}{2}-\frac{1 \cdot 3}{2}=4,5$.

В общем случае площадь треугольника равна $\frac{a \cdot h}{2} \pm \frac{b \cdot h}{2}=\frac{(a \pm b) \cdot h}{2}$, причем заметим, что $a \pm b$ - сторона нашего треугольника, т.е. мы получили правильную формулу.

г), д) Опишем вокруг данного треугольника прямоугольник (рис.7, г,д). Заметим, что площадь треугольника получается так: вычитаем из площади прямоугольника, площади всех лишних кусочков, которые могут быть прямоугольными треугольниками (случай г) или прямоугольными треугольниками и прямоугольниками (случай д). Вычислим эти площади. Случай г): $6 \cdot 5-\frac{2 \cdot 4}{2}-\frac{2 \cdot 5}{2}-\frac{3 \cdot 6}{2}=12$, 
случай д): $6 \cdot 5-1 \cdot 2-\frac{2 \cdot 4}{2}-\frac{1 \cdot 4}{2}-\frac{5 \cdot 6}{2}=7$.

\section{Задачи для самостоятельного решения}

5. На клетчатой бумаге проведен отрезок $A B$, не проходящий по сторонам клеток. Постройте наибольшее число равнобедренных треугольников $A B C$ с основанием $A C$. Решите задачу для двух отрезков, изображенных на рисунке 8.

6. На клетчатой бумаге проведен отрезок $A B$, не проходящий по сторонам клеток. Постройте угол $A B C$, равный $45^{\circ}$.

7. На клетчатой бумаге в ее узлах отмечены две точки. Постройте квадрат, для которого данные две точки являются противоположными вершинами.

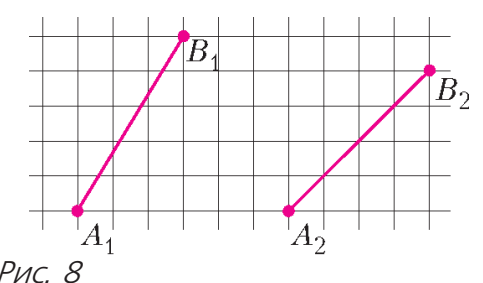

8. а) Опираясь на построение предыдущей задачи, постройте серединный перпендикуляр к данному отрезку. б) Как построить центр окружности, описанной вокруг данного треугольника?

9. Нарисуйте квадрат с вершинами в узлах сетки площадью: а) 10; б) 17; в) 26 клеток.

\section{КОНКУРС ИМЕНИ А.П.САВИНА}

Мы продолжаем очередной конкурс по решению математических задач. Они рассчитаны в первую очередь на учащихся 7-9 классов, но мы будем рады участию школьников всех возрастов. Конкурс проводится совместно с журналом “Квантик».

Высылайте решения задач, с которыми справитесь, электронной почтой по адресу: savin.contest@gmail.com или обычной почтой по адресу: 119296 Москва, Ленинский проспект, 64-A, “Квант» (с пометкой “Конкурс имени А.П.Савина»). Кроме имени и фамилии укажите город, школу и класс, в котором вы учитесь, а также обратный почтовый адрес.

Мы приветствуем участие в конкурсе не только отдельных школьников, но и команд (в таком случае присылается одна работа со списком участников). Участвовать можно, начиная с любого тура. Победителей ждут дипломы журнала "Квант» и призы. Задания, решения и результаты публикуются на сайте sites.google.com/view/savin-contest

\section{Желаем успеха!}

9. Электронные часы показывают часы и минуты. Вася подошел к часам и заметил, что сейчас на них палиндром - время выглядит как АВ:ВА. Он решил подождать, когда это повторится, но, просидев 4 часа, так и не увидел второго палиндрома. А сколько ему еще осталось ждать?

$$
\text { Ю.Маркелов }
$$

10. Докажите, что любое целое число, не меньшее 12, можно записать как сумму двух составных чисел.

\section{И.Митрофанов}

11. На столе лежит картонный треугольник $A B C$. C ним несколько раз делают следующую операцию. Выбирают его вершину, обозначим ее $X$, переворачивают треугольник и кладут его так, чтобы точка $X$ попала на том же место, что и раньше, и угол $X$ треугольника совпал со своим предыдущим положением. Обозначим такую операцию $P_{X}$. Докажите, что после последовательности операций $P_{A}, P_{B}, P_{C}$, $P_{A}, P_{B}, P_{C}$ треугольник займет первоначальное положение.

\section{B.Paсторгуев}

12. В клетки прямоугольника $2 \times n$ записывают числа $1,2, \ldots, 2 n$ (в каждую клетку - по одному числу). Сначала в левую нижнюю угловую клетку записывают число 1, затем в одну из соседних клеток - число 2 , потом в одну из соседних с ранее занятыми - 3 и т.д.; все последующие числа записывают в клетки, соседние с ранее занятыми. Докажите, что существует ровно $2^{n-1} n$ ! способов заполнить таблицу.

B.Pасторгуев 